\title{
Análise crítica da formulação do Morosil e sua comercialização no tratamento da
}

\section{obesidade}

Critical analysis of the formulation of Morosil and its commercialization in the treatment of obesity

Análisis crítico de la formulación de Morosil y su comercialización en el tratamiento de la obesidad

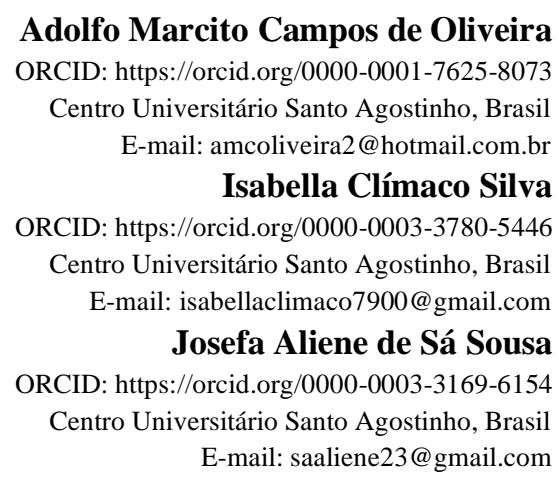

\section{Resumo}

Os tratamentos com fitoterápicos tem ganhado força nos últimos anos, principalmente para o tratamento da obesidade, porém existem poucas evidências científicas sobre os mesmos. Dessa forma o presente estudo teve como objetivo realizar uma análise crítica da sua comercialização e eficácia no tratamento da obesidade. A pesquisa foi realizada em farmácias de manipulação e drogarias da cidade de Teresina - PI, por meio de uma análise observacional, foram avaliadas as informações técnicas dos produtos que tem como princípio ativo o Toranja Citrussinensis (morosil). Além disso também será realizado uma busca de artigos nas seguintes bases de dados Science Direct, PubMed e MEDLINE. Em uma tabela foram descritas as especificações das formulações de morosil comercializadas em Farmácias de manipulação de Teresina-PI, onde majoritariamente são comercializados na apresentação de cápsulas, com exceção da farmácia $C$ tipo 2 que dispõe também da apresentação sachê. Todas as formulações possuem uma dosagem de 500mg, com exceção da Farmácia E tipo 1, consequentemente, todas têm posologia de 1 vez ao dia, exceto a Farmácia E tipo 1 que tem posologia de 2 vezes ao dia. Todos possuem venda livre. Os preços entre as Farmácias variam de 188R \$ à 90R \$, tendo na Farmácia D as opções do morosil associado a outros componentes ou de forma isolada. Diante do exposto, é possível inferir as informações contidas nas formulações magistrais dos produtos manipulados são essencialmente importantes para garantir uma boa adesão farmacológica, minimizar os potenciais efeitos adversos, garantir a segurança e a eficácia.

Palavras-chave: Morosil; Fitoterapia; Obesidade.

\begin{abstract}
Herbal treatments have gained strength in recent years, mainly for the treatment of obesity, but there is little scientific evidence about them. Thus, the present study aimed to conduct a critical analysis of its commercialization and effectiveness in the treatment of obesity. The research was carried out in manipulation pharmacies and drugstores in the city of Teresina - PI, through an observational analysis, the technical information of the products whose active ingredient is the Grapefruit Citrussinensis (morosil) was analyzed. In addition, a search for articles will also be carried out in the following databases Science Direct, PubMed and MEDLINE. The table below shows the specifications of the morosil formulations sold in pharmacies in Teresina-PI, where they are mostly sold in the presentation of capsules, with the exception of pharmacy $\mathrm{C}$ type 2, which also has the sachet presentation. All formulations have a dosage of 500mg, with the exception of Pharmacy E type 1, consequently, all have a dosage once a day, except Pharmacy E type 1 which has a dosage twice a day. All have free sales. Prices between pharmacies range from $188 \mathrm{R} \$$ to $90 \mathrm{R} \$$, with Pharmacy D having the morosil options associated with other components or in
\end{abstract}


isolation. Given the above, it is possible to infer the information contained in the masterful formulations of the products handled are essentially important to ensure good pharmacological adherence, minimize potential adverse effects, ensure safety and efficacy.

Keywords: Morosil; Phytotherapy; Obesity.

\section{Resumen}

Los tratamientos a base de hierbas han ganado fuerza en los últimos años, principalmente para el tratamiento de la obesidad, pero existe poca evidencia científica al respecto. Así, el presente estudio tuvo como objetivo realizar un análisis crítico de su comercialización y efectividad en el tratamiento de la obesidad. La investigación se realizó en farmacias de manipulación y droguerías de la ciudad de Teresina - PI, mediante un análisis observacional, se analizó la información técnica de los productos cuyo ingrediente activo es el Pomelo Citrussinensis (morosil). Además, también se realizará una búsqueda de artículos en las siguientes bases de datos Science Direct, PubMed y MEDLINE. La siguiente tabla muestra las especificaciones de las formulaciones de morosil comercializadas en las farmacias de Teresina-PI, donde se comercializan mayoritariamente en presentación de cápsulas, a excepción de la farmacia tipo 2 , que también tiene la presentación de sobre. Todas las formulaciones tienen una dosis de $500 \mathrm{mg}$, a excepción de la Farmacia E tipo 1, en consecuencia, todas tienen una dosis una vez al día, excepto la Farmacia E tipo 1 que tiene una dosis dos veces al día. Todos tienen ventas gratuitas. Los precios entre las farmacias oscilan entre $188 \mathrm{R} \$$ y $90 \mathrm{R} \$$, y la Farmacia D tiene las opciones de morosil asociadas con otros componentes o de forma aislada. Dado lo anterior, es posible inferir que la información contenida en las magistrales formulaciones de los productos que se manejan son de fundamental importancia para asegurar una buena adherencia farmacológica, minimizar los posibles efectos adversos, garantizar la seguridad y eficacia.

Palabras clave: Morosil; Fitoterapia; Obesidad.

\section{Introdução}

Atualmente a obesidade é um dos mais graves problemas de saúde pública no mundo, caracterizada pelo acúmulo excessivo de gordura corporal no indivíduo, a mesma geralmente está associada a ingestão exagerado de calorias e o menor gasto energético, por essa razão ela também se relaciona aos distúrbios metabólicos, associado principalmente com mau controle sobre o comportamento alimentar, gerando assim um aumento de massa corporal de gordura no organismo (Sousa; Santos; Rocha, 2019). Segundo a Organização Mundial da Saúde (OMS, 2008) a obesidade é considerada como uma epidemia mundial condicionada principalmente pelo perfil alimentar e de atividade física. A prevalência atribuída vem sendo diversos processos biopsicossociais, em que o "ambiente" (político, econômico, social, cultural), e não apenas o indivíduo e suas escolhas, assume um lugar estratégico na análise do problema e nas propostas de intervenções.

Contudo, parte dos desafios reside em compreender como esses múltiplos fatores interagem. É de grande importância que a seja reconhecida a obesidade como um problema de saúde pública, podendo ser iniciada em qualquer idade e caracterizada como uma doença de origem. Quando a perda de calorias ultrapassa o consumo de energia, acumula-se gordura nas células, levando à obesidade. Se o corpo excede o peso ideal da pessoa, a solução para a obesidade é limitar a ingestão de calorias e, aos poucos, elevar o gasto de energia (Campana, 2012). De acordo com Guyton (2003, p.815), "o tratamento da obesidade significa simplesmente em limitar a entrada de energia a um valor menor do que a energia gasta". Isso expressa que o tratamento da obesidade tem por objetivo um acréscimo de jejum diminuindo o apetite com medicamentos ou técnicas de emagrecimento com ajuda de terapias estéticas.

Os tratamentos com fitoterápicos tem ganhado força nos últimos anos, principalmente para o tratamento da obesidade, mas existem poucas evidências científicas sobre os mesmos. É de suma importância melhorar a qualificação do profissional de saúde para o manejo dos fitoterápicos. Sendo relevante o conhecimento do uso dos fitoterápicos, pois tratamentos, devidamente acompanhados por profissionais juntamente com atividades físicas equilibrada, apresenta efeitos comprovados na perda de peso (Fetrow, 2000). Os fitoterápicos possuem consequentemente aplicações consagradas pelo conhecimento popular 
sendo utilizados em grande escala pela população como primeiro recurso terapêutico devido ao fácil acesso, baixo custo, na ausência de efeitos colaterais não há necessidade de prescrição médica. O uso de medicamentos fitoterápicos deve ter o acompanhamento por um profissional capacitado, a fim de individualizar o uso para cada paciente em especifico, evitando os possíveis efeitos adversos e proporcionando maiores benefícios para a saúde do paciente com o uso racional e consciente do medicamento (Albertasse; Thomaz; Andrade, 2010).

O extrato seco do suco de laranja vermelha ou do fruto conhecido como (Toranja Citrussinensis) é comercializado com o nome comercial de Morosil, este tem sido aproveitado no tratamento da obesidade. Este tipo de laranja originária da Itália foi gerado pela mutação no cultivo de laranjas pigmentadas dando sua cor característica, o vermelho intenso devido ao elevado teor de antocianinas (Tsuda et al., 2006). De acordo com Cardile et al. (2015), o extrato seco do suco de laranja vermelha detém substâncias ativas que agem no metabolismo dos adipócitos (são células que estabelecem a temperatura do corpo humano e são responsáveis por incluir lipídeos e a gordura ingerida) que com ajuda de flavonóides e outras substâncias reduzem a massa corporal pela ação molecular específica que causam. (Cardile et al., 2015).

O extrato da laranja vermelha possui baixas calorias, mas estão cobertos de nutrientes sendo uma excelente fonte de vitaminas A e C, com índice glicêmico de 25, propondo que não afeta níveis de açúcar e insulina no sangue (Ware, 2015). Dessa forma espera-se mostrar uma crítica da comercialização do morosil, bem como avaliar sua eficácia no tratamento da obesidade com base na literatura, além de mostrar como estava à disposição do morosil que estava à venda nas farmácias tanto de manipulação, como nas drogarias, comparação dos dados obtidos com as informações técnicas disponíveis, verificação da sua eficácia para o tratamento da obesidade na literatura e assim apresentar um estudo base nas principais funcionalidades do morosil a fim de driblar a obesidade por meio da busca em artigos científicos.

\section{Metodologia}

\section{Tipo de pesquisa}

O presente trabalho, trata-se de uma pesquisa de campo com caráter descritivo e abordagem quantitativa. A pesquisa de campo foi realizada em farmácias de manipulação, avaliando a disposição dos produtos que utilizam morosil Segundo Trivinõs (1987), o estudo descritivo exige do pesquisador uma delimitação precisa de técnicas, métodos, modelos e teorias que orientarão a coleta e interpretação de dados, cujo objetivo é conferir validade científica a pesquisa. A população e a amostra também foram delimitadas, assim como os objetivos, os termos, as variáveis, as hipóteses e as questões de pesquisa. Para Fontelles et al. (2009), a abordagem quantitativa, diz respeito aos dados coletados, estes são expressos sob forma de dados numéricos, empregando-se recursos e técnicas estatísticas para classifica-los e analisá-los.

\section{Cenário e participantes do estudo}

A pesquisa foi realizada em farmácias de manipulação e drogarias da cidade de Teresina - PI, onde, por meio de uma análise observacional, foram avaliadas as informações técnicas disponíveis (como são vendidos, formulações, embalagens e bulas) dos produtos que tem como princípio ativo o morosil. Além disso, também foi realizada uma busca de artigos nas seguintes bases de dados: Science Direct, PubMed e MEDLINE utilizando como descritores "Morosil"; "Laranja Vermelha"; "Fitoterapia". Foarm incluídos artigos da faixa temporal de 2015 a 2020, que estavam no idioma português e inglês e que tratam da temática em questão. Foram excluídos artigos incompletos e comunicações rápidas. Ainda foi analisada a legislação dos fitoterápicos e as farmacopeias disponíveis. 


\section{Coleta de dados}

Os dados foram coletados através uma analisa observacional, serão analisadas as informações técnicas dos produtos que tem como princípio ativo o morosil. Foi avaliada a disposição desse produto de acordo com o local que estão sendo comercializados, comparando com as informações técnicas disponíveis e confortando os dados com a literatura.

\section{Análise de dados}

Os resultados obtidos foram organizados através do Software Microsoft ${ }^{\circ}$ Excel® 2010 e com tabulação através de tabelas e gráficos para discussão.

\section{Procedimentos éticos}

Por tratar-se de análise de dados que não envolvem pacientes (seres humanos e animais) que utiliza apenas informações técnicas não haverá necessidade de apreciação em Comitê de Ética em Pesquisa (CEP), pois este não provocou intervenção nas pessoas estudadas, estando de acordo com a pesquisa a resolução de número 466 de 12 de dezembro de 2012 do Conselho Nacional de Saúde (CNS), que trata das Diretrizes e Normas Regulamentadoras de Pesquisas Envolvendo Seres Humanos.

\section{Resultados}

$\mathrm{Na}$ Tabela 1 são mostradas as especificações das formulações de morosil comercializadas em Farmácias de manipulação de Teresina-PI, onde majoritariamente são comercializados na apresentação de cápsulas, com exceção da farmácia $\mathrm{C}$ tipo 2 que dispõe também da apresentação sachê. Todas as formulações possuem uma dosagem de 500mg, com exceção da Farmácia E tipo 1, consequentemente, todas têm posologia de 1 vez ao dia, exceto a Farmácia E tipo 1 que tem posologia de 2 vezes ao dia. Todos possuem venda livre. Os preços entre as Farmácias variam de $188 \$$ à $90 \$$, tendo na Farmácia D as opções do morosil associado a outros componentes ou de forma isolada.

Tabela 1. Caracterização crítica da formulação do morosil comercializados em farmácias de Teresina-PI.

\begin{tabular}{|c|c|c|c|c|c|}
\hline Farmácias & Preço & $\begin{array}{c}\text { Forma } \\
\text { farmacêutica }\end{array}$ & Dosagem & Posologia & Prescrição \\
\hline Farmácia A & $138 \mathrm{R} \$$ & Cápsulas & $500 \mathrm{mg}$ & $24 \mathrm{~h} \mathrm{em} 24 \mathrm{~h}$ & Venda livre \\
\hline Farmácia B & 90R\$ & Cápsulas & $500 \mathrm{mg}$ & $24 \mathrm{~h} \mathrm{em} 24 \mathrm{~h}$ & Venda livre \\
\hline Farmácia $\mathrm{C}$ tipo 1 & $95 \mathrm{R} \$$ & Cápsulas & $500 \mathrm{mg}$ & $24 \mathrm{~h} \mathrm{em} 24 \mathrm{~h}$ & Venda livre \\
\hline Farmácia C tipo 2 & $101 \mathrm{RR} \$$ & Sachê & $500 \mathrm{mg}$ & $24 \mathrm{~h}$ em $24 \mathrm{~h}$ & Venda livre \\
\hline Farmácia D tipo 1 & $\begin{array}{c}120 \mathrm{R} \$ \\
\text { (associado) }\end{array}$ & Cápsulas & $500 \mathrm{mg}$ & $24 \mathrm{~h}$ em $24 \mathrm{~h}$ & Venda livre \\
\hline Farmácia D tipo 2 & 110R\$ (simples) & Cápsulas & $500 \mathrm{mg}$ & $24 \mathrm{~h}$ em $24 \mathrm{~h}$ & Venda livre \\
\hline Farmácia E tipo 1 & $188 \mathrm{R} \$$ & Cápsulas & $250 \mathrm{mg}$ & $12 \mathrm{~h} \mathrm{em} 12 \mathrm{~h}$ & Venda livre \\
\hline Farmácia E tipo 2 & $120 \mathrm{R} \$$ & Cápsulas & $500 \mathrm{mg}$ & $24 \mathrm{~h}$ em $24 \mathrm{~h}$ & Venda livre \\
\hline
\end{tabular}




\section{Discussão}

Toranja citrussinensis, vulgarmente conhecida como laranja amarga, é comumente empregada como um agente aromatizante e acidificante para alimentos. Além do óleo essencial e seus constituintes, os frutos de T. citrussinensis são fontes de compostos do tipo flavonóide com inúmeros efeitos biológicos. Ademais, os flavonóides glicosídeos têm sido isolados da planta e os conteúdos de amina biogênica e flavanona foram definidos. Ultimamente, os fitoterápicos têm evidenciado um papel de destaque na descoberta de novos medicamentos (Teixeira \& Dos Santos, 2019).

Normalmente, os fitofármacos são produtos de venda livre, isto é, não necessita de prescrição, contribuindo assim, diretamente à automedicação e orientação farmacêutica. Para que a automedicação seja racional e de forma correta é preciso que a informação que chega ao consumidor seja facilmente compreensível, contendo dados sobre dosagem, forma farmacêutica que favoreça a adesão, e sua posologia. Além disso, é imprescindível um preço condizente à aos padrões socioeconômicos no qual as farmácias (Tomasello et al., 2019).

Além disso, o morosil é um ativo extraído da laranja vermelha mouro, cultivada no mediterrâneo, um excelente antioxidante, com elevada concentração de vitamina $\mathrm{C}$ e ácidos hidroxicinâmicos. Além disso, contém antocianina C3G, uma grande aliada na diminuição de medidas e no gerenciamento do peso, corrobora a diminuição da gordura abdominal de 25 a $50 \%$ e favorece para a atenuação da esteatose hepática. O morosil possui uma dosagem usual de $250 \mathrm{mg}$ a $500 \mathrm{mg}$ ao dia (Akhlaghi et al., 2011).

A dosagem e a posologia de uma formulação são trabalhadas de forma complementar, onde ambas andam juntas, pois a posologia, que é o intervalo de tempo onde uma dose e outra é tomada, é calculada a partir do tempo de meia vida que a dosagem tomada diminui pela metade e, assim, necessita de uma nova dosagem, caracterizando assim sua posologia (Leite et al., 2008). Por este motivo, as dosagens apresentadas são proporcionais às posologias mostradas na Tabela acima. Deste modo, é garantida a melhor resposta terapêutica do fármaco em uso, diluindo os riscos de efeitos adversos resultantes de superdosagem e minimiza também as possibilidades de os pacientes disporem de uma subdosagem (Karimi, Oskoueian, Hendra, Oskoueian, \& Jaafar, 2012).

Em adição, na literatura encontra-se uma prevalência de estudos mostrando a ingestão de produtos fitoterápicos, sobretudo, por meio da apresentação farmacêutica de cápsulas, como por exemplo, relatado por Morais et al., 2019, onde nesse estudo, ele mostra que 53,17\% dos fitoterápicos usados em seu estudo foram empregados na forma farmacêutica em cápsula, corroborando ao evidenciado nesse estudo. Numa pesquisa, delegado por Guimarães et al., 2014, que também mostrou que dos 744 medicamentos fitoterápicos dispensados na farmácia magistral analisada, dispuseram-se 14 formas farmacêuticas diferentes, contudo, a cápsula evidenciou como a forma farmacêutica mais frequentemente empregada em formulações magistrais, totalizando $59 \%$.

Além disso, em outro estudo, as apresentações farmacêuticas magistrais, foram encontradas apenas as formas de cápsulas gelatinosas $(98 \%)$ e sachês $(2 \%)$, resultado que corrobora com este trabalho realizado com o fitoterápico morosil, em que houve a prevalência de cápsulas gelatinosas na manipulação de fórmulas magistrais. Os tipos de cápsulas analisadas embora tenham as dosagens iguais e serem geradas das mesmas partes da planta (extrato seco da casca) e ausentes de excipientes, ainda demonstram diferenças quanto ao número da cápsula usada. Essa diferença pode estar atrelada as variações na densidade compactada das outras amostras (Jaisin et al., 2020).

Portanto, o uso de cápsulas como forma farmacêutica requer que algumas circunstâncias sejam atendidas para sua viabilização, cujo quais se destacam a estabilidade ideal do fármaco quando encapsulado, a liberação ideal do conteúdo, incluindo o fármaco e excipientes, nos líquidos gastrointestinais, a isenção do efeito biológico dos excipientes presentes na formulação, a ausência de gosto ou odor desagradável dos componentes após a encapsulação, o tamanho adequado do 
invólucro para cada formulação, reduzida variabilidade de dose, independentemente do tamanho de lote produzido, em escala industrial ou artesanal e a compatibilidade física e química entre o fármaco, os excipientes e o envoltório em condições ambientais normais (Stohs, Preuss, \& Shara, 2011).

Diante disso, é importante ressaltar que a manipulação de formulações magistrais, necessita de uma seleção minuciosa e sistemática de princípios ativos e da dose para garantir a eficácia terapêutica esperada. Ademais, a farmácia de manipulação procura trabalhar em conjunto com os médicos, ao ofertar informações relevantes de cada fármaco (Kostopoulou, Therios, Roumeliotis, Kanellis, \& Molassiotis, 2015). A fórmula manipulada valoriza a prescrição profissional, pois torna melhor a relação médico-paciente e concede uma firmeza da fórmula para o paciente precisa mais especificamente, como pessoa única e singular em relação aos sintomas que sente e que nem sempre se acostuma a formulações definidas previamente (Moraes et al., 2009).

Alguns medicamentos não se fazem necessário uma receita médica, desse modo, consumidor tem a oportunidade de obter medicamentos como laxantes, diuréticos e fórmulas para controle do peso. Existem diferentes tipos de medicamentos fitoterápicos no mercado, sendo uma parte deles com registro simplificado, de venda livre sem prescrição médica, como castanha da Índia, Aloe vera, centela-asiática, alcachofra, morosil, camomila; e outros com tarja vermelha, no qual sua comercialização se faz necessário uma prescrição médica, como urva-ursina, cimicífuga, equinácea, Gingko biloba, hipérico, kava-kava e valeriana (Pimenta et al., 2016).

As prescrições de fitoterápicos emagrecedores também são feitas por outros profissionais, como mostrado em estudo, onde se constatou que durante três meses as prescrições alcançaram a uma média 92,17\%. O mesmo acontece na avaliação mensal, em que a quantidade de prescrições de emagrecedores chega a até 99,87\%. Embora a busca seja prevalente para os emagrecedores, os fitofármacos podem ser utilizados para outros diferentes fins, como ansiolíticos, orexígenos e redutores de colesterol (Wang et al., 2014).

Contudo, existe uma relação grande entre os fitoterápicos dispensados por uma farmácia de a uma prescrição profissional, além da automedicação (sem prescrição). Para os fitofármacos com formulação simplificada em relação a frequência de dispensação, por meio de uma prescrição de um profissional de saúde, foi de 56\% e sem prescrição foi de $44 \%$. Já os fitoterápicos compostos, como evidenciado na tabela acima, a frequência de dispensação, através de prescrição e sem prescrição, foi de 50\% para ambos (Yang et al., 2010). O farmacêutico, também é um profissional da saúde muito importante, e possui a função de orientar os pacientes em todos os aspectos do tratamento da obesidade, sobretudo o que está relacionado aos medicamentos que faz uso. $\mathrm{O}$ uso de medicamentos para o controle da obesidade tem de ser feito com cuidado, e é imprescindível que sejam utilizados somente em momentos especiais conforme o julgamento sistemático do médico assistente (Rahnama et al., 2015).

As "fórmulas emagrecedoras" são prescritas por médicos, em geral endocrinologistas, contudo, pode acontecer de aparecer receitas prescritas por outras especialidades como clínico geral, ginecologistas ou até mesmo dermatologistas. O grande motivo pela grande fé das pessoas nas fórmulas é o fato de delas crerem que a fórmula foi minuciosamente dosada para seus casos e de sempre conhecerem uma pessoa que perdeu peso ao tomá-la (Namazi et al., 2014). De fato, há pessoas que perdem peso e perdem dez quilos no início do tratamento, porém, depois disso, a perda fica progressivamente lenta, devido a termogênese adaptativa que o organismo exerce. Por este motivo, as formulações são consideradas exemplos de polifarmácia. Desse modo, é extremamente importante todas essas informações sobre a comercialização do morosil, uma vez que são amplamente empregados para o tratamento da obesidade e também para obter o conhecimento sobre as variações das características que o morosil possui (Sarrou, Chatzopoulou, Dimassi-Theriou, \& Therios, 2013). 


\section{Conclusão}

Diante do exposto, é possível inferir as informações contidas nas formulações magistrais dos produtos manipulados são essencialmente importantes para garantir uma boa adesão farmacológica, além de minimizar os potenciais efeitos adversos e, consequentemente, garantir uma segurança e eficácia adequada, uma vez que a grande maioria não dispõe necessariamente de uma prescrição de um profissional de saúde habilitado, visando o uso racional do medicamento. Além disso, o preço do produto magistral é importante, de modo que se encaixe dentro do padrão social da população no qual está inserida, uma vez que o morosil é um fitoterápico utilizado para o emagrecimento que tem um mercado bastantemente aquecido.

\section{Referências}

Akhlaghi, M., Shabanian, G., Rafieian-Kopaei, M., Parvin, N., Saadat, M., \& Akhlaghi, M. (2011). Flor de Citrus aurantium e ansiedade pré-operatória. Revista Brasileira de Anestesiologia, 61(6), 707-712. https://doi.org/10.1590/s0034-70942011000600002

Guimarães, G. P., Reis, M. Y. de F. A., da Silva, D. T. C., Mendonça Junior, F. J. B., Converti, A., Pessoa, A., \& da Silva, J. A. (2014). Antifungal activity of topical microemulsion containing a thiophene derivative. Brazilian Journal of Microbiology, 45(2), 545-550. https://doi.org/10.1590/S151783822014000200024.

Jaisin, Y., Ratanachamnong, P., Wongsawatkul, O., Watthammawut, A., Malaniyom, K., \& Natewong, S. (2020). Antioxidant and anti-inflammatory effects of piperine on UV-B-irradiated human HaCaT keratinocyte cells. Life Sciences, 263, 118607. https://doi.org/10.1016/j.lfs.2020.118607

Karimi, E., Oskoueian, E., Hendra, R., Oskoueian, A., \& Jaafar, H. Z. E. (2012). Phenolic Compounds Characterization and Biological Activities of Citrus aurantium Bloom. Molecules, 17(2), 1203-1218. https://doi.org/10.3390/molecules17021203

Kostopoulou, Z., Therios, I., Roumeliotis, E., Kanellis, A. K., \& Molassiotis, A. (2015). Melatonin combined with ascorbic acid provides salt adaptation in Citrus aurantium L. seedlings. Plant Physiology and Biochemistry, 86, 155-165. https://doi.org/10.1016/j.plaphy.2014.11.021

Leite, M. P., Fassin, J., Baziloni, E. M. F., Almeida, R. N., Mattei, R., \& Leite, J. R. (2008). Behavioral effects of essential oil of Citrus aurantium L. inhalation in rats. Revista Brasileira de Farmacognosia, 18(SUPPL.), 661-666. https://doi.org/10.1590/S0102-695X2008000500003

Moraes, T. M., Kushima, H., Moleiro, F. C., Santos, R. C., Machado Rocha, L. R., Marques, M. O., \& Hiruma-Lima, C. A. (2009). Effects of limonene and essential oil from Citrus aurantium on gastric mucosa: Role of prostaglandins and gastric mucus secretion. Chemico-Biological Interactions, 180(3), 499-505. https://doi.org/10.1016/j.cbi.2009.04.006

Morais, T. L., Neves, C. A., Borges, N. C., Lima, A. M. V., Silva, L. F. L., Castro, L. T. S., \& Cunha, P. H. J. (2019). Evaluation of tear production, intraocular pressure, retinography and ocular biometry in bovine Nelore and Gir breeds. Arquivo Brasileiro de Medicina Veterinaria e Zootecnia, 71(1), 177186. https://doi.org/10.1590/1678-4162-10263

Namazi, M., Ali Akbari, S. A., Mojab, F., Talebi, A., Majd, H. A., \& Jannesari, S. (2014). Effects of citrus Aurantium (bitter orange) on the severity of firststage labor pain. Iranian Journal of Pharmaceutical Research, 13(3), 1011-1018. https://doi.org/10.22037/ijpr.2014.1553

Pimenta, F. C. F., Alves, M. F., Pimenta, M. B. F., Melo, S. A. L., Almeida, A. A. F. de, Leite, J. R., \& Almeida, R. N. de. (2016). Anxiolytic Effect of Citrus aurantium L. on Patients with Chronic Myeloid Leukemia. Phytotherapy Research, 30(4), 613-617. https://doi.org/10.1002/ptr.5566

Rahnama, S., Rabiei, Z., Alibabaei, Z., Mokhtari, S., Rafieian-kopaei, M., \& Deris, F. (2015). Anti-amnesic activity of Citrus aurantium flowers extract against scopolamine-induced memory impairments in rats. Neurological Sciences, 36(4), 553-560. https://doi.org/10.1007/s10072-014-1991-2

Sarrou, E., Chatzopoulou, P., Dimassi-Theriou, K., \& Therios, I. (2013). Volatile Constituents and Antioxidant Activity of Peel, Flowers and Leaf Oils of Citrus aurantium L. Growing in Greece. Molecules, 18(9), 10639-10647. https://doi.org/10.3390/molecules180910639

Stohs, S. J., Preuss, H. G., \& Shara, M. (2011). The Safety of Citrus aurantium (Bitter Orange) and its Primary Protoalkaloid $p$-Synephrine. Phytotherapy Research, 25(10), 1421-1428. https://doi.org/10.1002/ptr.3490

Teixeira, F. O., \& Dos Santos, S. R. (2019). Métodos Estatísticos Aplicados Na Análise Do Fitoterápico Morosil. In Simpósio de Engenharia de Produção (Vol. 0). Retrieved from http://www.dep.uem.br/gdct/index.php/simeprod/article/view/1725

Tomasello, B., Malfa, G. A., La Mantia, A., Miceli, N., Sferrazzo, G., Taviano, M. F., \& Acquaviva, R. (2019). Anti-adipogenic and anti-oxidant effects of a standardised extract of Moro blood oranges (Citrus sinensis (L.) Osbeck) during adipocyte differentiation of 3T3-L1 preadipocytes. Natural Product Research. https://doi.org/10.1080/14786419.2019.1660337

Wang, Q. H., Shu, Z. P., Xu, B. Q., Xing, N., Jiao, W. J., Yang, B. Y., \& Kuang, H. X. (2014). Structural characterization and antioxidant activities of 
Research, Society and Development, v. 10, n. 6, e16910615616, 2021

(CC BY 4.0) | ISSN 2525-3409 | DOI: http://dx.doi.org/10.33448/rsd-v10i6.15616

polysaccharides from Citrus aurantium L. International Journal of Biological Macromolecules, 67, 112-123. https://doi.org/10.1016/j.ijbiomac.2014.03.004

Yang, L., Cao, Y.-L., Jiang, J.-G., Lin, Q.-S., Chen, J., \& Zhu, L. (2010). Response surface optimization of ultrasound-assisted flavonoids extraction from the flower of Citrus aurantium L. var. amara Engl. Journal of Separation Science, 33(9), 1349-1355. https://doi.org/10.1002/jssc.200900776 